Chapter 5

\title{
Bacterial Interaction with Graphene Particles and Surfaces
}

\author{
Shannon M. Notley, Russell J. Crawford and \\ Elena P. Ivanova \\ Additional information is available at the end of the chapter \\ http://dx.doi.org/10.5772/56172
}

\section{Introduction}

Research into the properties and uses of graphene has rapidly expanded over the past decade. Indeed, prior to the seminal paper by Geim and Novoselov in 2004 [1] which eventually led to the 2009 Nobel Prize for physics, the potential of this material was relatively underappreciated. Graphene is a monolayer thick, two dimensional form of carbon atoms linked together in a hexagonal lattice. The sp2 hybridisation of all bonds across the sheet gives rise to its interesting and unique, physical, mechanical, thermal and electrical properties. Thus graphene can be considered to be a 2 dimensional form of its analogue graphite [2]. Importantly, the properties of graphene vary significantly to the bulk material graphite, particularly in terms of electron mobility, and these significant feature differences have driven research in fields as diverse as electronics, materials, energy, defence, security, water and health $[3,4]$.

Much of the focus of graphene research has been in high value added electronics where miniaturization is a key driver. However to date, the bulk of the material that is produced is geared toward research applications or in composites production. The use of graphene as the load bearing component in composites is highly beneficial [5]. Whilst compatibilization with matrix remains an issue, the high tensile strength of graphene coupled with the ultra-high aspect ratios that are possible using most particulate production methods has led to the rapid uptake of this technology. Furthermore, the potential for using graphene in thin film and coatings applications is increasing, with the goal to produce modified surfaces with improved structural integrity, better heat resistance, conductance, transparence or protection from corrosion [6, 7]. Recent studies have also shown that graphene could also be used in water purification applications due to its high cation exchange capacity and vast available surface area [8]. Other potential applications of graphene include in sensor technology, opto-electronic 
devices, high electron mobility transistors, supercapacitors, catalysis, photovoltaic (nanocrystal solar cell) and desalination.

Many of these uses for graphene will have significant economic and environmental benefits however it is of great importance that the possible downside effects of incorporating graphene into products which may come into contact with the biosphere are accurately known. A thorough understanding of the interaction with biological material is essential prior to the uptake and utilisation of graphene on a wide scale, particularly if there is significant potential for it to find its way into the environment and human body. Potential biomedical applications for graphene have been suggested. The low surface energy of graphene makes it an attractive substrate for the delivery of hydrophobic drugs. Furthermore, the unique structure could allow its use as a contrast agent in biomedical imaging. The influence of particle size is important in biomedical applications. Single layer graphene may potentially sit in between the leaflets of a lipid bi-layer, it is unlikely though that few layer graphene however could be incorporated. Furthermore, the ability for cells to phagocytise graphene is largely dependent upon particle dimensions. Whilst cytotoxicity is hence of a clear and present concern, graphene as a building block presents a novel opportunity for designing and functionalising systems and products that can potentially transform the manner in which we live. Hence there is a strong push for understanding the interaction of graphene with cells and bacteria. Bacterial interaction with surfaces is ubiquitous in nature. There are also countless examples where biofilm formation leads to significant environmental and health problems. For instance in a marine environment, biofilm formation on ship hulls can lead to corrosion and increased drag as bacteria colonisation can lead to subsequent attachment of other organisms such as barnacles. Whilst this can result in a substantial economic cost, the health problems arising from bacteria attachment to medical implants can be fatal if not properly dealt with in a timely fashion.

This chapter discusses the current knowledge in the area of bacteria - graphene surfaces interactions. A number of graphene production approaches will be also considered along with the brief outline of the graphene surfaces characterisation techniques. A number of recent interesting studies will be reviewed, however at this early stage, no clear conclusions can be drawn on the toxicity of the graphene to bacteria, with some studies suggesting bactericidal activity whilst others suggesting inhibition of attachment and others still no anti-bacterial action. The reasons behind this lack of consistency are therefore of great importance to determine.

\section{Production methods and characterisation of graphene}

It is useful at this point to define more precisely the term "graphene". The pristine form, as shown in the schematic diagram in Figure 1, contains complete sp2 hybridisation with no defects and only a single layer of atoms. However, in the literature graphene may also refer to few layered material held together through van der Waals forces as well as graphene oxide which is subsequently reduced. It should be noted here, reduced graphene oxide has a significant number of defects which heavily impacts electron mobility and gives rise to a non- 
flat (puckered) structure. Few layered graphene also has some of the advantages of graphene such as high aspect ratio yet does not have the same optical and electrical properties of the single layer material.

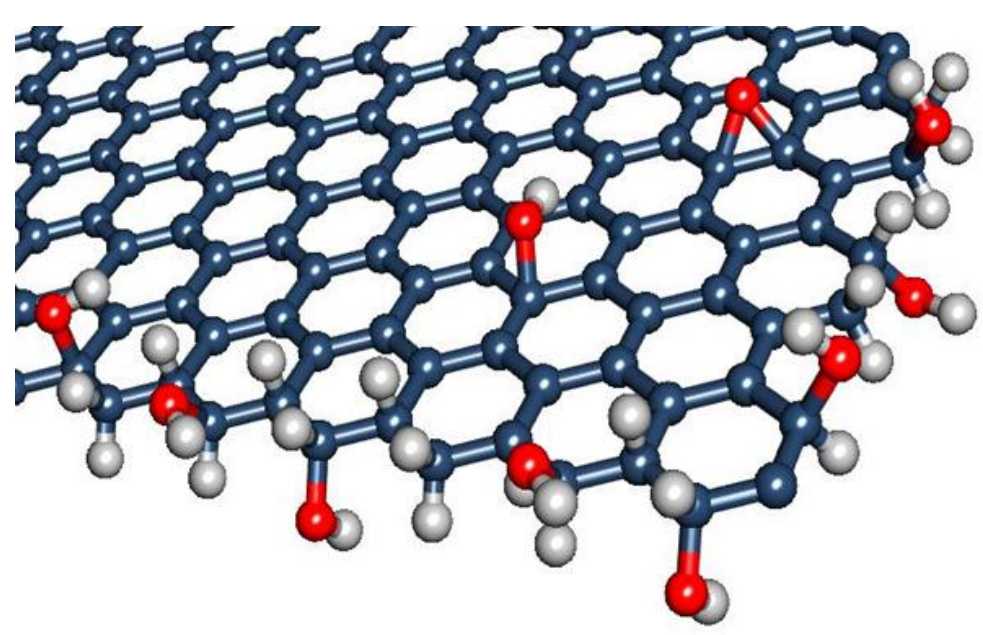

Figure 1. Schematic diagram depicting single layer "pristine" graphene with edge defects (carbon atoms in blue, oxygen in red and hydrogen in white).

It should be apparent that it is not possible for the $C$ atoms at the edges of the particles to be sp2 hybridised. The edges are stabilised through a number of chemical functionalities including epoxy, hydroxyl and carboxylic groups, the latter giving rise to charge in liquids of high dielectric constant such as water. Thus, the smaller the particles, the higher the ratio of edge groups and therefore charges per unit area. Clearly, there are significant differences in the physico-chemical properties of graphene which is influenced ultimately by the production method, these are described below. It is hence not surprising then that the interaction with bacteria would be influenced also by the source of the graphene.

The physical and chemical properties of graphene particles are typically characterised using a variety of techniques including microscopy and spectroscopy. Transmission electron microscopy (TEM) and atomic force microscopy (AFM) imaging are employed to determine the dimensions of the particles. TEM is useful as it can show multiple layers through contrast variation; single layer sheets are relatively transparent to the electron beam however its main advantage is showing the lateral dimensions. AFM is also a technique which has been employed which can give the thickness of the particles. The graphene sheets are typically attached to a substrate prior to imaging, an offset is usually found corresponding to a trapped vapour layer under the particles however for stepped few layer graphene samples, the thickness between layers agrees with literature values. One of the greater advantages of AFM is the ability to combine with Raman spectroscopy to show unequivocally the number of layers of graphene. 
Spectroscopic means for characterising graphene are routinely used to give molecular scale chemical properties. Techniques such as Raman and UV-Vis spectroscopy are most common. Raman spectroscopy is considered the gold standard technique for determining the number of graphene layers as well as the presence of any defects [9]. Figure 2 shows a typical Raman spectrum for exfoliated graphene particles as well as graphite, the most prominent peaks are those at $1335 \mathrm{~cm}^{-1}, 1565 \mathrm{~cm}^{-1}$ and $2675 \mathrm{~cm}^{-1}$ which are termed the D, G and 2D (2D has sometimes been erroneously termed $\left.G^{\prime}\right)$. The $G$ peak at $1565 \mathrm{~cm}^{-1}$ shown in both spectra corresponds to sp2 hybridised carbon and is relatively invariant upon exfoliation. The peak at $1335 \mathrm{~cm}^{1}$ is sp3 carbon and is introduced through bond cleavage and hence indicates the presence of either edge or basal plane defects. The most important peak though is in the region of $2675 \mathrm{~cm}^{-1}$ where the position and shape indicate the number of graphene layers. UV-Vis spectrophotometry is also quite useful in determining the presence of defects and has often been used to probe the extent of reduction of graphene oxide to graphene. Strong absorbance across the spectrum indicates a highly conjugated structure and furthermore, the wavelength of intensity maximum provides evidence for the presence of defects.
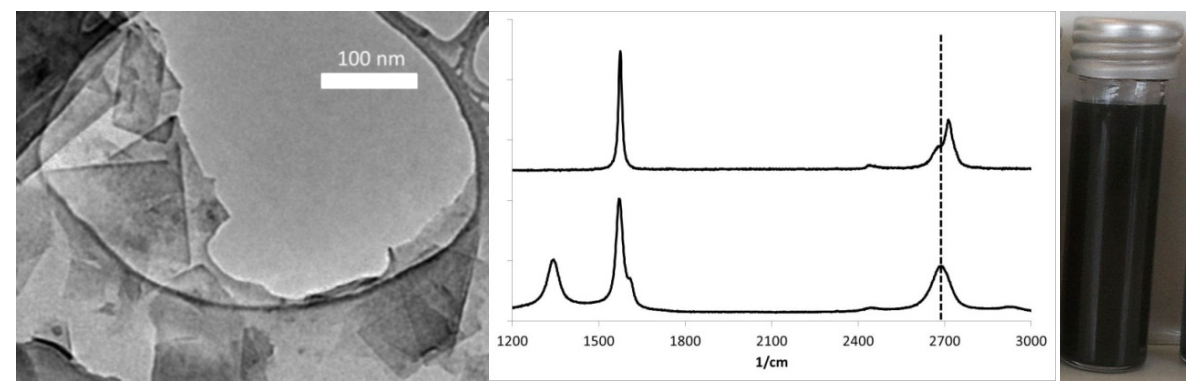

Figure 2. Left: TEM of graphene sheets, middle: Raman spectra of single layer graphene and bulk graphite, right: aqueous suspension of graphene (Reproduced with permission from Reference 30).

The characterisation of graphene is important as it aids in the discussion of differences in the properties depending on preparation method. The most common methods, pertinent to studies involving the interaction of graphene with bacteria are presented below, along with the physical and chemical properties. These include exfoliation of graphite as well the reduction of dispersed graphite oxide and vapour deposition techniques.

\subsection{Mechanical exfoliation}

This is often referred to as the "Scotch Tape Method" where single and few layer sheets of graphene are removed from a bulk graphite sample through attachment to adhesive tape. This mechanical exfoliation of sheets is considered to be an excellent route to micrometre to submillimetre sized graphene particles without the introduction of any defects (apart from those at the edges). Thus, the sheets produced using this method are considered "pristine" and hence have excellent electrical conductivity. Graphene prepared through mechanical exfoliation is used mainly in research applications where low quantity, high purity samples are required. 
Most often, the samples are fixed to a supporting substrate exposing the conjugated basal planes. Thus, the surface for bacterial study is "atomically" smooth with lateral dimensions in excess of the typical size of a bacteria cell.

\subsection{Epitaxial growth and Chemical Vapour Deposition (CVD)}

Both techniques of epitaxial growth and chemical vapour deposition rely on a suitable substrate for supporting the deposited graphene [10]. Typically these substrates are metal or metal-like (eg silicon) as process parameters usually involve high temperatures precluding deposition onto polymer surfaces. Epitaxial growth of graphene was first demonstrated on a silicon carbide substrate [11-13]. Temperatures in excess of $1300^{\circ} \mathrm{C}$ and ultra-high vacuum are normally required to produce a graphene multilayered materials through sublimation of silicon, usually with thicknesses in the range of 5-100 sheets but over large (wafer scale) areas. However some studies have had success with moderate vacuum conditions in controlled environments. Epitaxial growth of graphene shows great promise as lithographic techniques may be used in conjunction to produce patterned surfaces. It should be noted though, that epitaxially grown graphene has significantly different properties to the exfoliated material which is largely due to interfacial effects of the underlying SiC substrate. For example, the quantum Hall effect is absent with epitaxial graphene and furthermore, slight differences in periodicity and electron diffraction patterns are observed. Thus electron mobility is not of the same order as exfoliated graphene.

Aside from $\mathrm{SiC}$ substrates, other materials such as transition metals have been employed to produce graphene layers. CVD has been employed with iridium [14], ruthenium [15], platinum and nickel [16]. Nucleation and growth occurs after exposure of the substrate to a hydrocarbon gas such as methane or ethylene at low pressures (often ultra high vacuum conditions) and high temperatures. The condensed gas is converted to a carbon layer upon cooling of the substrate. This typically produces a multilayered graphene surface, the properties of which are similar to those detailed for epitaxially grown graphene.

Both techniques of epitaxially growth and CVD are capable of producing large area graphene with high transparency and conductance which are suitable for studies of bacteria. They also have the added advantage of ease of patterning which can be beneficial for potential investigations of directed growth of cells [16]. There is control though over the thickness (ie number of layers) which is also advantageous and the roughness of such graphene surfaces is somewhat conformal to the underlying substrate [17].

\subsection{Reduced Graphene Oxide (RGO)}

One of the most common processes for producing industrial scale quantities of graphene is based upon a method first developed by Hummers for oxidising graphite [18]. Briefly, the modified Hummers method involves the oxidation of graphite powder with for example, sulphuric acid and potassium permanganate to produce graphite oxide. This product is then dispersed in water and subjected to sonication to split apart the bulk material into single and few layer graphene oxide $[5,19,20]$. The suspension is relatively stable due to the high charge 
(zeta potential greater than $-60 \mathrm{mV}$ ) on the exfoliated graphene oxide sheets. Subsequently, the graphene oxide is reduced, often with hydrazine to re-form the $\mathrm{C}-\mathrm{C}$ bonds with most, but importantly not all of the conjugation maintained. Whilst possible to produce on an industrial scale, the Hummers method does have some significant drawbacks including the highly toxic nature of some reactants as well as high cost of oxidisers. Furthermore there is a somewhat complicated post-production purification treatment. However one of the greatest disadvantages is the introduction of a significant level of defects which reduces the conductivity of the particles substantially as well as creating a degree of roughness [10]. Hence RGO is most often used in composites applications where conductivity is not as important and roughness increases the surface area. In terms of potential interaction with bacteria, the reduced conductivity and increased roughness are not necessarily detrimental and studies involving graphene oxide and RGO have been reported.

A similar procedure using carbon nanotubes as the starting material has also been reported which can result in the production of graphene. Often termed "unzipping", carbon nanotubes have been treated with strong oxidizers to cleave bonds allowing the tube to unroll. The product is subsequently reduced to repair defects and regenerate the sheet conjugation. The graphene particles produced from unzipping of nanotubes are hence of limited dimensions which also means they have an inherently larger edge defect ratio and charge per unit area than other preparation techniques.

\subsection{Liquid phase exfoliation}

One promising technique for the production of relatively chemically unmodified graphene in large quantities is liquid phase exfoliation, sometimes in the presence of surfactants and polymers [21-32]. This method for the preparation of graphene has been performed in water, organic solvents as well as ionic liquids [33, 34] however most often the concentration produced is low $(<1 \mathrm{mg} / \mathrm{mL})$. Typically, the interfacial energy of the liquid phase is matched to the surface energy of graphite promoting exfoliation under ultrasonication. In other words, the cohesive energy of the liquid phase and solid phase are comparable. This is achieved with appropriate choice of organic solvent, for example, N-methylpyrrolidone (NMP), N,NDimethylacetamide (DMA), $\gamma$-butyrolactone (GBL) and 1,3-dimethyl-2-imidazolidinone (DMEU) or ionic liquid with a surface tension of the order of about $41 \mathrm{~mJ} / \mathrm{m}^{2}$. In aqueous suspension, surface tension is reduced to the optimum energy range through the addition of a surfactant in order to separate the graphene sheets beyond the range of the strong attractive van der Waals forces. The surfactant also has a secondary role in preventing re-aggregation through adsorption to the graphene surface resulting in a repulsive energy between sheets.

A variety of surfactants have been employed in aqueous solution processing through sonication exfoliation. Monomeric surfactants, typically with cationic charge have been extensively used. Through adsorption, the graphene produced in this manner has a significant positive charge preventing re-aggregation. The surfactant is not adsorbed irreversibly so changes in solution conditions (eg through dialysis or dilution) can result in de-stabilisation of the suspensions. Polymeric type surfactants such as Pluronics have also been used which tend to improve stability due to the strongly bound nature of the hydrophobic components [30]. 
Clearly, the presence of any bound surfactant to the surface of graphene produced in this manner will have a significant influence on the interaction with bacteria. Furthermore, depending on processing parameters such as sonication power, sonication time, presence of salt and surfactants and centrifugation, the particles produced can be chemically varied. High sonication power leads to cavitation which can cleave bonds and oxidise the sheets as well as introduce defects. However, gentle sonication gives relatively pristine sheets suitable for both composites and electronics applications. Centrifugation is also important to separate single and few layer graphene from the unexfoliated bulk material. Importantly, liquid phase exfoliation always produces a distribution of graphene thickness and hence careful control of sedimentation is required to maximise the proportion of single layer graphene. Sample thickness may also influence the interaction with bacteria as will be discussed in later sections.

\subsection{Surfaces prepared from graphene particles}

Graphene surfaces have been prepared in a number of ways using particles as a starting material. These include "paper" like surfaces, supported and free standing, thin films deposited from solution onto a substrate as well as the development of thicker interfaces using the layer-by-layer technique. These methods are described in greater detail below.

One convenient method for producing macroscopically large areas of graphene oxide and graphene is to form a "paper" [20,35]. Typically, a suspension of the particles is vacuum or pressured filtered, the directional flow gives rise to the alignment of particles to form a lamella structure as demonstrated by $\mathrm{x}$-ray diffraction. Upon drying of this graphene particulate filter cake, the attractive forces between the sheets leads to a very strong material which can be peeled away from the underlying membrane substrate. The resultant material is often referred to as paper as the process is very similar to that involving wood fibres. In contrast though to conventional paper surfaces, graphene paper has a higher tensile strength and stiffness. The alignment of the particles means that the surface topography is flat and furthermore, the thickness of the carbon film can be tuned simply by increasing the concentration of graphene in the suspension prior to filtering. Many studies have presented cross-sectional images of the paper using SEM where the lamella structure is confirmed. Depending on thickness of the graphene paper, such cross-sections could also be used for bacterial studies. It is also possible to create hybrid paper structures through reactions within water layers in the graphene film or through simple addition of other materials [36, 37].

The direct deposition of graphene particles onto a planar surface has also been extensively used to create areas of graphene suitable for further studies. Attempts have been made to "stitch" together such particles however many of the important properties such as high conductivity were lost. Thus, in most cases, a mosaic like structure of single and few layer graphene particles is present. Imaging of these surfaces using AFM profiling, combined with Raman spectroscopy is arguably the best way of characterising the graphene particles but it should also be apparent that these type of surfaces present a convenient way of producing graphene modified surfaces. Depending on the source of such particles, the areas covered by graphene is variable but is usually of the order a square micron. 
In order to achieve a complete coverage, the layer-by-layer technique [38, 39] has been used. This involves the alternate deposition of positively and negatively charged components, using polyelectrolytes, however with a native negative charge, graphene oxide and graphene with edge defects have been introduced into such assemblies [20, 29, 40]. The thickness of the film containing graphene can be easily selected by determining the desired number of bilayers. Figure 3 shows the structure of the multilayer assembly incorporating cationically stabilised graphene and poly(acrylic acid) as a function of the number of layers [29].

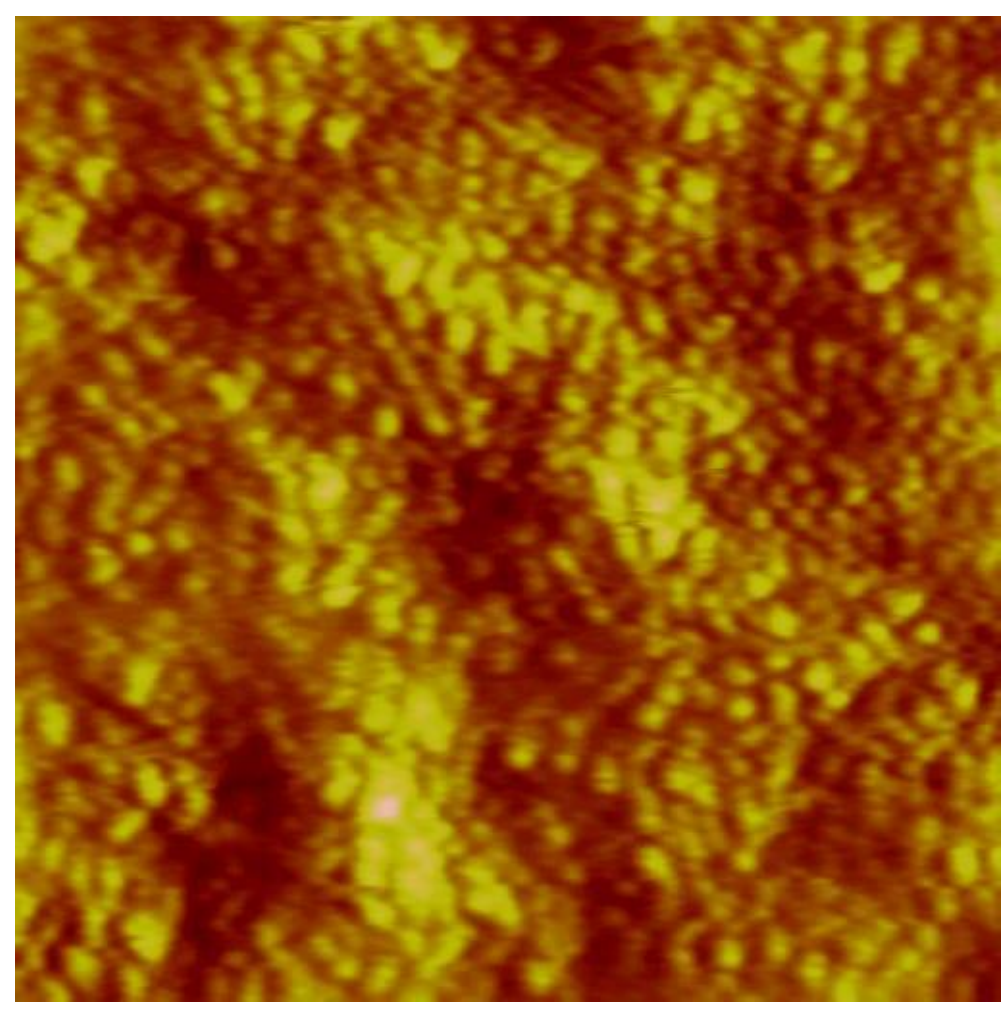

Figure 3. Surface structure of a graphene thin film prepared through the alternate deposition of cationically modified graphene and polyacrylic acid probed using atomic force microscopy imaging, the scale is $500 \mathrm{~nm} \times 500 \mathrm{~nm}$. (Reproduced with permission from Reference 29).

\section{Some insights on the nature of bacterial attachment to surfaces}

In a thermodynamic sense, adhesion can be defined as the energy required to create new surface area upon separation in a defined medium (such as air or water). As this suggests, the effective surface energies of the two interfaces are hence of critical importance. Thus the surface 
chemistry of the bacteria as well as the substrata to which the bacteria may attach must be well understood and considered as one of the possible parameters that may either promote or inhibit bacterial attachment and subsequent biofilm formation. The physical surface structure is also a critical factor influencing the interaction with bacteria. These details are discussed further below.

The chemistry of the substrate has been shown to influence the adhesion of bacteria and the build-up of the biofilm. Typically, bacteria have shown greater affinity for hydrophobic surfaces [41]. This has been hypothesised to be due to hydrophobic interactions although this is certainly dependent on the type of bacteria studied. Furthermore, an effective strategy for the creation of anti-bacterial surfaces has been to modify the surface to give a hydrophilic chemistry with polymeric materials such as polyethylene glycol (PEG) which are highly hydrated. In order for attachment to occur, the water molecules must be displaced, which is a thermodynamically unfavourable result. At high ionic strengths, the PEG chains can dehydrate and the efficacy for preventing bacterial adhesion of such surfaces is weaker [42]. It is commonly believed that adsorption on hydrophobic surfaces is rapid with strong binding forces, while adhesion to hydrophilic surfaces followed the model of reversible and irreversible adhesion proposed by Marshall [43] and can be described by DLVO theory (Derjaguin, Landau, Verwey, and Overbeek) [44, 45]. Initially, a weak and reversible stage of the adhesion was observed at separation distances of several nanometers, at which point the bacterium can be removed by shear forces or desorb spontaneously. At a later stage, this attachment can be converted into irreversible adhesion by synthesis of extracellular biopolymers or by stabilization of conformational changes in existing polymers. These polymers bridge separation distances of less than $1 \mathrm{~nm}$, displacing the adsorbed water and /or neutralising the electrostatic repulsion. Another factor that may influence the adhesion of bacteria is the presence of surface charge. Many surfaces carry a significant net charge due to ion dissolution, ion adsorption or surface ionisation, the latter effect gives rise to the potential for either net positive or net negative charge depending on $\mathrm{pH}$. This tends to be less important for polymeric surfaces which are inherently hydrophobic. It is interesting to note that graphene, in a similar sense to graphite has an intermediate wettability and depending on the method of production, may have a varying level of charge.

At this point, it is useful to introduce some definitions to make a distinction between surfaces which inhibit attachment and surfaces which effectively destroy bacteria on contact [46]. Both types of surfaces are often classed as "anti-bacterial" however the latter should more correctly be classed as bactericidal. Some chemistries such as those involving quaternary ammonium groups have shown strong bactericidal action through inducing permeability of the membrane. It has been demonstrated that surface morphology can also be used to kill bacteria. Typically, such structured surfaces have significant roughness or peaks with high aspect ratios which can cause the bacterial cell wall to rupture under its own weight upon contact [46]. Clearly in the case of graphene, the edges also fulfil this latter criterion however the basal planes can be considered atomically smooth. Therefore there is an expectation then the interaction of bacteria with graphene will be highly dependent on the orientation of the surface relative to the bacteria. 


\subsection{Surface chemistry of bacterial cells}

The bacterial cell outer layer is composed of complex biological molecules; therefore at the time of the cell-surface interaction the cell surface chemistry is heterogeneous and depends on various physicochemical and biological parameters. For example it was shown that on the surface of the cyanobacterium Calothrix sp. KC97 that the three main functional groups (carboxyl, phosphoryl, and amine) present are present in several discrete binding sites [47]. Carboxyl groups are found in abundance in cell wall constituents such as diaminopimelic and glutamic acids in the peptide cross-linkages of peptidoglycan molecules; or carboxylated teichoic acids, covalently bound to peptidoglycan, or other phosphoryl-containing polymers in the cell envelope which include LPS. Lipids present in the cell wall may also exhibit phosphoryl groups of the phosphatidylglycerol and phosphatidylcholine. It was also reported that the negatively charged carboxyl and positively charged amine groups, present at natural $\mathrm{pH}$, usually occur in approximately equal proportions. The slight increase in the negative charge with increasing $\mathrm{pH}$ was suggested to be predominantly a result of deprotonation of carboxylic and phosphoric groups to form negatively charged species but may also be contributed to by deprotonation of amine groups to form electroneutral species.

In addition to the inherent heterogeneity of cellular outer layers, bacteria secrete a variety of extracellular polymeric substances (EPS), including polysaccharides, proteins, and nucleic acids, that vary in molecular mass and structural properties [48]. Extracellular polysaccharides (EPSs) are present in many forms, including capsular polysaccharides, which are also referred to as 'cell-bound extracellular polymeric substances' which remain connected to cell surfaces by means of a covalent attachment to phospholipid or lipid A molecules present at the bacterial surface [49]. While some capsular polysaccharides may be released into the growth medium (i.e. become 'free') as a consequence of the low stability of the phosphodiester linkage between the polysaccharide and the phospholipid membrane anchor, other tightly attached capsular polysaccharides form a distinct structural layer (the capsule) which encloses the cell and serves as a protective layer. This layer acts as a shield on the cell surface, affording the cell protection from major bacterial pathogens [50]. Most significantly, EPS play a major role in mediating the bacterial colonization of surfaces, biotic and abiotic, by enabling cell adhesion and co-aggregation via dipole interactions, covalent or ionic bonding, steric interactions, and hydrophobic association, making the target surface more attractive for bacterial attachment. The temperature, solution $\mathrm{pH}$, electrolyte and macromolecule concentration, and adsorbent surface chemistry will directly influence the chemical composition and structure of the EPS substances that are responsible for the surface conditioning.

\subsection{Interaction of bacteria with "graphene" modified surfaces}

Only a relative few studies have investigated the interaction of bacteria with graphene or graphene-like surfaces in comparison to other materials such as minerals, metals and plastics. The unique structure of graphene, in particular the physical dimensions which are characterised by the high aspect ratio, provide opportunities for creating surfaces with tailored properties which may inhibit or promote bacterial attachment as well as potentially inducing cell death. The varied preparation procedures for producing single and few layer graphene 
materials also provide the ability to tune the interaction with bacteria. These are perhaps the reasons why there is no consistent picture regarding the interaction of bacteria with such surfaces modified in this way [51]. However, there are clear circumstances where the buildup of the biofilm is inhibited. The available studies are summarised below.

There is great interest in producing graphene modified surfaces for anti-bacterial applications. Studies have suggested that such surfaces are indeed anti-bacterial yet others show the promotion of cell attachment. The first study undertaken for the investigation of bacterial interaction with a graphene-like surface was by Akhavan and Ghaderi in 2010 that used Gramnegative (E. coli) and Gram-positive (S. aureus) bacteria [52]. In this study, single and few layer graphene oxide and reduced graphene oxide deposited onto a stainless steel substrate was used. The orientation of particles was such that a significant number of edges were exposed. A substantial loss in cell viability for both E. coli and S. aureus was observed. However within this investigation, some trends were reported. For example, the cell viability was less for the Gram positive bacteria S. aureus and furthermore, the reduced graphene oxide surface had a greater ability to inhibit attachment as well as kill the bacteria. This study showed that membrane damage induced through contact with the graphene oxide and reduced graphene oxide particles occurred through measurement of the efflux of the cytoplasmic materials which supports the observation of a greater toxicity toward Gram-positive bacteria. The authors suggested in addition that the reduced graphene oxide particles have sharper edges than the oxidated counterparts leading to increased potency however this theory is not well supported by the direct imaging of particles, nor the extensive literature in this area [1,2].

$\mathrm{Hu}$ et al also investigated the interaction of E. coli with graphene oxide and reduced graphene oxide surfaces prepared through vacuum filtration into a paper like surface [53]. In this instance, the particles lay relatively flat on the surface with only a few edges oriented away from the interface. An airborne bacteria test was used in this study with the viability of cells subsequently determined. Similar to the work of Akhavan and Ghaderi, a significant loss of viability was observed for both graphene oxide and reduced graphene oxide; however in contrast they determined the greatest loss for the graphene oxide. Again, the toxicity mechanism was suggested to be due to membrane damage induced through contact with the particulate edges which was confirmed through scanning electron microscopy imaging.

In contrast to the above mentioned studies where graphene oxide has shown anti-bacterial activity, the study of Ruiz et al suggested that there is no detrimental effect to bacteria and indeed that bacterial growth rates were higher [54]. Graphene oxide paper surfaces were used in this study, the material prepared using a modified Hummers method together with E. coli as the model bacteria. Growth rates of bacteria were up to 3 times as fast on membranes modified with a high surface coverage of graphene oxide with a preferential attachment to areas with high particle density. No high resolution images of the paper surface were presented however it is likely that using this vacuum filtration preparation procedure that the graphene oxide particles are lying flat on the membrane substrate with few exposed edges. Previously it has been suggested that the cytotoxicity is due to membrane damage caused by rupture due to particle edges [51]. The oxidised basal planes which are however exposed in this case may 
confer an increased wettability on the particles which in turn allows a stronger interaction with the bacterial LPS through hydrogen bonding.

The interaction of bacteria with composite surfaces of graphene materials and other polymers has also been investigated. Many exfoliation techniques including the Hummers method and liquid phase exfoliation lend themselves to easy modification of the surface properties of the graphene particles through binding with polymers in either a physisorbed or chemisorbed sense [28]. This is due to the introduction of reactive sites or more simply charged groups which may lead to electrostatic adsorption of oppositely charged (cationic) polyelectrolytes. The combination with inherently anti-bacterial polymers such as those containing quaternary ammonium groups has proven highly useful. Sreeprasad et al produced composite films of graphene oxide (and reduced graphene oxide) and lactoferrin or chitosan [55]. E. coli was used as the bacteria in this study. Without surface modification through protein or polymer adsorption, the anti-bacterial activity of graphene oxide and reduced graphene oxide was weak. A significant increase in cytotoxicity was observed through incorporation of lactoferrin however the greatest loss in cell viability was due to the combination of lactoferrin and chitosan. There was no statistical difference in the graphene material observed.

Another study involving graphene oxide composite films with included polyvinyl-Ncarbazole, a polymer with inherent antibacterial characteristics [56, 57]. The efficacy of these composite surfaces against two Gram-negative bacteria (E. coli and C. metallidurans) and two Gram-positive bacteria (B. subtilus and R. opacus) was investigated. The anti-bacterial activity of the composite material was higher than the unmodified graphene oxide particles and surfaces. Not only did the surfaces inhibit build-up of the biofilm but it also inactivated a significant proportion of bacteria. Again, there was a greater loss in viability of Gram-positive bacteria exposed to the composite film. This study also showed that whilst anti-bacterial activity was observed, no significant cytotoxicity toward eukaryotic cells (human fibroblast) was observed suggesting that graphene oxide-polyvinyl-N-carbazole composites could potentially be used in clinical applications.

More recently another graphene oxide-polymer composite material also showed anti-bacterial activity as well as the promotion of the growth of human cells (adipose-derived stem cells) [58]. Poly-l-lysine (PLL) is a cationic polyelectrolyte which can adsorb irreversibly to graphene oxide through an electrostatic interaction. PLL has been shown to have anti-microbial activity but has also been used for the immobilization of cells to negatively charged surfaces such as silica hence Some et al used functionalised graphene oxide with PLL to investigate cytotoxicity of E. coli. In addition to the graphene oxide composite, the particles were subsequently reduced and the anti-bacterial activity studied. This study showed that there was no anti-bacterial activity of unmodified graphene oxide, as with previous studies, the surfaces were essentially arranged in a flat conformation and hence edges were not exposed. However, the graphene oxide-PLL composite showed potent anti-bacterial activity toward the Gram-negative bacteria E. coli. The relatively highly cationically charged surface was speculated to be the mechanism inhibiting attachment. Some et al also modified the composite to boost the amount of cationic charges through covalent attachment of a diazonium salt which resulted in a decrease in cell adherence. Staining experiments demonstrated that cell death was also induced. 
In the current literature, all studies involving bacteria have been conducted with either graphene oxide or reduced graphene oxide particles which have subsequently been formed into a surface for attachment and viability studies. There is still no consensus view in regard to the inherent anti-bacterial nature of such surfaces. The information available suggests there is some difference toward cytotoxicity depending upon the orientation of the particles attached to the surface. Graphene oxide and reduced graphene oxide sheets with the sharp edges exposed to contact with the bacterial membrane seem to induce rupture. In contrast, the studies which have shown no anti-bacterial activity tend to involve surfaces with the basal planes at the interface. As outlined in the previous sections, the materials used to date in studying the interaction with bacteria are quite distinct from what could be called "pristine" graphene or graphene produced through epitaxial growth. That is, no studies involving the highly conjugated, defect free and single layer of sp2 hybridised carbon have been reported.

\section{Interaction of graphene particles with bacteria in suspension}

Many of the studies outlined above also investigated the interaction of bacteria with graphene oxide and reduced graphene oxide particles in suspension with comparable results to those involving substrates produced from these particles [54, 55, 58, 59]. These studies usually investigated cell viability as a function of time and in general terms, greater loss in viability was observed for Gram-positive bacteria. In all of these studies, membrane rupture was proposed to be the toxicity mechanism. In suspension, the orientation of the graphene oxide or reduced graphene oxide particles may interact with bacteria in a manner of edgewise contact. Undoubtedly the evidence supports the suggestion of membrane rupture inducing cytotoxicity; however no molecular scale mechanism has yet been proposed.

In addition to the studies reported in the previous section, there have been others which have investigated the cytotoxicity of graphene and similar materials toward a range of bacteria in suspension. Such studies are highly important as there are many potential uses where graphene and graphene oxide have been proposed to be employed from clinical applications to water purification and coatings. In these uses it could reasonably be expected that the particles may interact with a range of cellular materials including bacteria. A recent study chronicled the antibacterial activity of a family of related materials dispersions including graphite, graphite oxide, graphene oxide and reduced graphene oxide toward the model Gram-negative bacteria E. coli [59]. All of these materials showed some anti-bacterial activity using a colony counting method. The materials with smallest thickness, that is graphene oxide and reduced graphene oxide showed a greater cytotoxicity with a preference for the reduced form. These authors noted that membrane rupture occurred however also demonstrated that oxidative stress was also a potential mechanism for the observed cell death.

The antibacterial efficiency of reduced graphene oxide nanosheets was also studied by Krishnamoorthy et al [60]. The authors studied the minimum inhibitory concentration (MIC) of the particles against both Gram-positive (E. faecalis and B. subtilis) and Gram-negative (E. coli and S. typhimurium) bacteria. The MIC of reduced graphene oxide particles was observed 
to be significantly lower for the Gram-negative bacteria. This was hypothesised to be due to the much thinner peptidoglycan layer of these types of bacteria. This is in direct contrast to other studies which suggest that the presence of the secondary cell membrane of Gramnegative bacteria provides a better resistance to membrane induced damage in the presence of these particles. The authors also provided evidence that enhanced lipid peroxidation occurred in suspensions containing reduced graphene oxide.

Many studies have investigated the difference in activity between graphene oxide and reduced graphene oxide yet to date only one has looked into the particle size dependence [61]. Liu et al demonstrated that larger sheets had a stronger antibacterial action than smaller graphene oxide particles. Different sized sheets were prepared through varying the sonication time in the modified Hummers method used to prepare the graphene oxide suspension. The lateral size difference was up to 2 orders of magnitude. The loss of viability of E. coli cells increased with increasing size and was due to the ability of larger sheets to effectively isolate and wrap bacterium cells preventing proliferation.

Akhavan et al have investigated the potential of "wrapping" of bacteria with graphene particles in order to reduce bioactivity [62]. The model Gram-negative bacterium E. coli was used in this study. The authors observed no significant inactivation of the bacteria in the presence of graphene oxide suspension or reduced form of graphene. However, the study was also conducted in the presence of melatonin, a reductant, which resulted in the aggregation of bacteria and functionalised graphene particles. AFM showed that the bacteria were ensconced by particles which resulted in a decrease in active cells.

Additional work by Akhavan and Ghaderi has demonstrated that E. coli was able to reduce graphene oxide under acidic conditions [63]. The oxygen containing functional groups were reduced by as much as $60 \%$ over a period of two days as the graphene oxide particles were supposed to be biocompatible. Interestingly, upon conversion to reduced graphene oxide, antibacterial action was shown, due in large part to the existing attached bacteria to the graphene surfaces as well as growth inhibition of these particles as previously discussed.

Another successful strategy that has been employed for producing increased anti-bacterial action of graphene oxide has been to functionalise the surfaces with nanometric silver particles. Zhang et al used gelatin as a "green" reducing agent for $\mathrm{Ag}^{2+}$ ions to form silver nanoparticles of size about $100 \mathrm{~nm}$ [64]. These were also stabilized with gelatin and subsequently mixed with a graphene oxide suspension. The positively charged amino groups of the gelatin stabilised silver particles were then electrostatically attracted to the negatively charge groups on the graphene oxide surface. These composite particles showed concentration dependence for the growth inhibition of E. coli with as little as 10 ppm required to reduce growth by $99.9 \%$ (the corresponding non-functionalised graphene oxide particles reduced growth by $38 \%$ ). Combining graphene oxide with silver nanoparticles was also undertaken by Das et al to investigate the interaction with the Gram-negative bacteria E. coli and P. aeruginosa [65]. Sodium borohydride as a reducing agent was added to a suspension of graphene oxide particles in the presence of $\mathrm{AgNO}_{3}$ under vigorous stirring to produce decorated sheets. The size of the Ag nanoparticles was of the order of $5-25 \mathrm{~nm}$. The authors showed an increasing growth delay of both bacterial strains with increasing nanoparticle concentration. 
A variation on the surface modification of graphene oxide sheets with nanoparticles is to create a hybrid material which is photocatalytic. This approach was taken by Liu et al where the surface was functionalised with $\mathrm{Ag}_{3} \mathrm{PO}_{4}$ nanoparticles to create a material with strong absorbance in the visible region [66]. These particles show enhanced photocatalytic activity as demonstrated by the degradation of a model dye AO7 through the production of reactive oxygen species. The authors also observed strong antibacterial action against $E$. coli of the composite graphene oxide- $\mathrm{Ag}_{3} \mathrm{PO}_{4}$ to a similar extent of synthesised $\mathrm{Ag}_{3} \mathrm{PO}_{4}$ nanoparticles. The mechanism of action was suggested to be due to oxidative stress induced disinfection upon exposure to visible light.

\section{Outlook}

Whilst much work has been undertaken toward understanding the interaction of bacteria and graphene (and related materials) few clear unambiguous trends can currently be drawn on bacteria properties or physico-chemical properties of the particles. The overwhelming majority of studies have been conducted with graphene oxide prepared using the Hummers method or with the subsequently reduced graphene oxide particles. As discussed, many of the unique properties of graphene such as high electron mobility are significantly diminished upon oxidation and reduction. Hence in order to adequately state the bacterial interaction with graphene and related materials, further studies using differently sourced materials must be undertaken. Such studies should involve exfoliated graphene which retains the extended conjugation or alternatively, epitaxially grown graphene materials.

The orientation of the graphene sheets immobilised at the interface has a clear influence on the observed bactericidal action. Single layer graphene and graphene oxide have a thickness of about $0.3 \mathrm{~nm}$, or atomic thickness, and hence have been speculated to be able to rupture the cell membrane. This was shown with both Gram-positive and Gram-negative bacteria which suggest the ultrastructure of prokaryotic cell walls is not a factor in the observed bactericidal activity. Furthermore, the studies which had an orientation of particles parallel to the interface may have inhibited bacterial attachment in some cases however cell death was not evident. In addition, the lateral size of particles had an influence on cell viability which supports the idea that the edge density is important. Surfaces patterned to a greater extent, particular on the length scale of the size of the bacteria will improve understanding of the role of particle orientation. Many techniques are described in the literature which may allow a greater degree of control over the orientation of particle surfaces through lithography. Epitaxially grown graphene surfaces combined with photolithography may prove useful to achieve this control.

The bacteria species studied to date are predominantly common types such as E. coli or $P$. aeruginosa. The variety of bacteria available is vast with diverse properties. It would be of great interest to expand the knowledge of the interaction with graphene and graphene oxide materials beyond these systems to others of high importance in clinical applications such as S. aureus or those with substantially different surface chemistries including highly hydrophobic bacteria. The studies detailed above have also used bacteria with simple geometries such as spheres and rods but other more complex geometries are common. 
The majority of studies have focussed on anti-bacterial applications of graphene oxide and reduced graphene oxide derivatives. Whilst this is undoubtedly an important avenue of research, there are many applications where promoting bacterial growth on surfaces is advantageous. Controlling particle orientation as well combining with biocompatible polymer materials may lead to the potential for utilising graphene particles in biomedical applications.

The increased potential use of graphene, graphene oxide and reduced graphene oxide surfaces and particles in technology demands a thorough understanding of cytotoxicity. Nanoparticles are becoming more prevalent in biomedical applications. Examples include the use of particles in medical imaging. Another prominent example is the use of gold nanorods which may induce local heating once incorporated into tissue for novel anti-tumour applications. Porous nanoparticles have also been suggested for drug delviery. Bacteria form an important component of the biomass and important function in the environment. Hence it is timely and essential that all potential benefits and disadvantages of the use of this new class of materials are appreciated prior to the widespread uptake and use of graphene in novel applications.

\section{Acknowledgements}

SN acknowledges financial support from the Australian Research Council under the Future Fellowships scheme.

\section{Author details}

Shannon M. Notley*, Russell J. Crawford and Elena P. Ivanova

*Address all correspondence to: snotley@swin.edu.au

Faculty of Life and Social Sciences, Swinburne University of Technology, Hawthorn VIC, Australia

\section{References}

[1] Novoselov, K. S, et al. Electric field effect in atomically thin carbon films. Science, (2004). , 666.

[2] Novoselov, K. S, et al. Two-dimensional atomic crystals. Proc. Natl. Acad. Sci. USA, (2005). , 10451.

[3] Geim, A. K. Graphene: Status and prospects. Science, (2009). , 1530-1534.

[4] Geim, A. K, \& Novoselov, K. S. The rise of graphene. Nat. Mater., (2007). , 183. 
[5] Stankovich, S, et al. Graphene-based composite materials. Nature, (2006). , 282-286.

[6] Singh RamanR.K., et al., Protecting copper form electrochemical degradation by graphene coating. Carbon, (2012). , 4040-4045.

[7] Prasai, D, et al. Graphene: corrosion-inhibiting coating. ACS Nano, (2012). , 1102-1108.

[8] Li, D, \& Kaner, R. B. Graphene-based materials. Science, (2008).

[9] Ferrari, A. C, et al. Raman Spectrum of Graphene and Graphene Layers. Phys. Rev. Lett., (2006). , 187401.

[10] Allen, M. J, Tung, V. C, \& Kaner, R. B. Honeycomb carbon: A review of graphene. Chem. Rev., (2010). , 132-145.

[11] De Heer, W. A, et al. Epitaxial graphene. Solid State Communications, (2007). , 92-100.

[12] Berger, C, et al. Electronic confinement and coherence in patterned epitaxial graphene. Science, (2006). , 1191-1196.

[13] Berger, C, et al. Ultrathin epitaxial graphite: 2D electron gas properties and a route twoard graphene-based nanoelectronics. J. Phys. Chem. B, (2004). , 19912-19916.

[14] Diaye, N, et al. Structure of epitaxial graphene on $\operatorname{Ir}(111)$. New Journal of Physics, (2008). , 043033.

[15] De Parga, A. L. V, et al. Periodically rippled graphene: Growth and spatially resolved electronic structure. Phys. Rev. Lett., (2008). , 056807-056804.

[16] Reina, A, et al. Large are, few layer graphene films on arbitrary substrates by chemical vapour deposition. Nano Lett., (2009). , 30-35.

[17] Rasool, H. I, et al. Continuity of graphene on polycrystalline copper. Nano Lett., (2011). , 251-256.

[18] Hummers, W. S, \& Offeman, R. E. Preparation of graphitic oxide. J. Am. Chem. Soc, (1958). , 1339-1339.

[19] Stankovich, S, et al. STable aqueous dispersions of graphitic nanoplatelets via the reduction of exfoliated graphite oxide in the presence of poly(sodium-4-styrenesulfonate). J. Mater. Chem., (2006). , 155-158.

[20] Li, D, et al. Processable aqueous dispersions of graphene nanosheets. Nature Nanotechnology, (2008). , 101-105.

[21] Coleman, J. N, et al. Two-dimensional nanosheets produced by liquid exfoliation of layered materials. Science, (2011). , 568-571.

[22] Hernandez, Y, et al. Measurement of multicomponent solubility parameters for graphene facilitates solvent discovery. Langmuir, (2010). , 3208-3213. 
[23] Hernandez, $\mathrm{Y}$, et al. High-yield production of graphene by liquid-phase exfoliation of graphite. Nat. Nanotechnol., (2008). , 563.

[24] Lotya, M, et al. Liquid phase production of graphene by exfoliation of graphite in surfactant/water solutions. J. Am. Chem. Soc,, (2009). , 3611-3620.

[25] Lotya, M, et al. High-concentration, surfactant-stabilized graphene dispersions. ACS Nano, (2010). , 3155-3162.

[26] Smith, R. J, et al. Large-scale exfoliation of inorganic layered compounds in aqueous surfactant solutions. Adv. Mater., (2011). , 3944-3948.

[27] Smith, R. J, Lotya, M, \& Coleman, J. N. The importance of repulsive potential barriers for the dispersion of graphene using surfactants New Journal of Physics, (2010). p. art (125008)

[28] Griffith, A, \& Notley, S. M. pH dependent stability of graphene suspensions with adsorbed weakly ionisable cationic polyelectrolyte. J. Colloid Interface Sci., (2012). , 210-215.

[29] Notley, S. M. Adsorption of polyelectrolyte modified graphene to silica surfaces: monolayers and multilayers. J. Colloid Interface Sci., (2012). , 35-40.

[30] Notley, S. M. Highly concentrated aqueous suspensions of graphene through ultrasonic exfoliation with continuous surfactant addition. Langmuir, (2012). , 14110-14113.

[31] Seo, J, et al. High-concentration aqueous dispersions of graphene using nonionic, biocompatible block copolymers. J. Phys. Chem. Lett., (2011). , 1004-1008.

[32] Liang, Y. T, \& Hersam, M. C. Highly concentrated graphene solutions via polymer enhanced solvent exfoliation and iterative solvent exchange. J. Am. Chem. Soc, (2010). , 17661-17663.

[33] Nuvoli, D, et al. High concentration few-layer graphene sheets obtained by liquid phase exfoliation of graphite in ionic liquid. J. Mater. Chem., (2011). , 3428-3431.

[34] Wang, X, et al. Direct exfoliation of natural graphite into micrometre size few layers graphene sheets using ionic liquids. Chem. Comm., (2010). , 4487-4489.

[35] Dikin, D. A, et al. Preparation and characterization of graphene oxide paper. Nature, (2007). , 457-460.

[36] $\mathrm{Hu}, \mathrm{T}$, et al. Flexible free-standing graphene-TiO2 hybrid paper for use as lithium ion battery anode materials. Carbon, (2013). , 322-326.

[37] Liu, F, et al. Folded structure graphene paper for high performance electrode material. Adv. Mater., (2012). , 1089-1094.

[38] Decher, G. Fuzzy Nano-assemblies:Toward layered polymeric multicomposites. Science, (1997). , 1232-1237. 
[39] Notley, S. M, Eriksson, M, \& Wågberg, L. Visco-elastic and adhesive properties of adsorbed polyelectrolyte multilayers determined in situ with QCM-D and AFM measurements. J. Colloid Interface Sci., (2005). , 29-37.

[40] Szabo, T, Szeri, A, \& Dekany, I. Composite graphitic nanolayers prepared by self-assembly between finely dispersed graphite oxide and a cationic polymer. Carbon, (2005). , 87-94.

[41] Doyle, R. J, \& Rosenberg, M. Microbial cell surface hydrophobicity, in American Society for MIcrobiology(1990). Washington.

[42] Liu, G, et al. Protein resistance of (ethylene oxide)n monolayers at the air-water interface: effects of packing density and chain length. Phys. Chem. Chem. Phys., (2007). , 6073-6082.

[43] Marshall, K. C. Biofilms: an overview of bacterial adhesion, activitiy and control at surfaces. ASM News, (1992). , 202-207.

[44] Bruscher, H. J, \& Van Der Mei, H. C. Physico-chemical interactions in initial microbial adhesion and relevance for biofiom formation. J. Dent. Res., (1997). , 24-32.

[45] Weerkamp, A. H, Uyen, H. M, \& Busscher, H. J. Effect of zeta potential and surface energy on bacterial adhesion to uncoated and saliva coated human enamel and dentin. J. Dent. Res., (1998). , 1483-1487.

[46] Ivanova, E. P, et al. Natural bactericidal surfaces: mechanical rupture of Pseudomonas aeruginosa cells by cicada wings. Small, (2012). , 2489-2494.

[47] Phoenix, V. R, et al. Characterization and Implications of the Cell Surface Reactivity of Calothrix sp. Strain KC97. Appl. Environ. Microbiol., (2002). , 4827-4834.

[48] Nazarenko, E.L., R.J. Crawford, and E.P. Ivanova, The structural diversity of carbohydrate antigens of selected gram-negative marine bacteria. Marine Drugs, 2011. 9: p. 1914-1954

[49] Deng, L, et al. Characterization of the Linkage between the Type III Capsular Polysaccharide and the Bacterial Cell Wall of Group B Streptococcus. J. Biol. Chem., (2000). , 7497-7504.

[50] Dong, C, et al. Wza the translocon for E. coli capsular polysaccharides defines a new class of membrane protein. Nature, (2006). , 226-229.

[51] Jastrzebska, A. M, Kurtycz, P, \& Olszyna, A. R. Recent advances in graphene family materials toxicity investigations. J. Nanopart. Res., (2012). , 1320-1328.

[52] Akhavan, O, \& Ghaderi, E. Toxicity of graphene and graphene oxide nanowalls against bacteria. ACS Nano, (2010). , 5731-5736.

[53] $\mathrm{Hu}, \mathrm{W}$, et al. Graphene-based antibacterial paper. ACS Nano, (2010). , 4317-4323. 
[54] Ruiz, O. N, et al. Graphene oxide: A nonspecific enhancer of cellular growth. ACS Nano, (2011). , 8100-8107.

[55] Sreeprasad, T. S, et al. Transparent, luminescent, antibacterial and patternable film forming composites of graphene oxide/reduced graphene oxide. ACS Applied Materials and Interfaces, (2011). , 2643-2654.

[56] Mejias CarpioI.E., et al., Toxicity of a polymer-graphene oxide composite against bacterial planktonic cells, biofilms, and mammalian cells. Nanoscale, (2012). , 4746-4756.

[57] Santos, C. M, et al. Chem. Commun., (2011). , 8892.

[58] Some, S, et al. Dual functions of highly potent graphene derivatives-poly-l-lysine composites to inhibit bacteria and support human cells. ACS Nano, (2012). , 7151-7161.

[59] Liu, S, et al. Antibacterial activity of graphite, graphite oxide, graphene oxide and reduced graphene oxide: Membrane and oxidative stress. ACS Nano, (2011). , 6971-6980.

[60] Krishnamoorthy, K, et al. Antibacterial efficiency of graphene nanosheets against pathogenic bacteria via lipid peroxidation. J. Phys. Chem. C., (2012). , 17820-17827.

[61] Liu, S, et al. Lateral dimension-dependent antibacterial activity of graphene oxide sheets. Langmuir, (2012). , 12364-12372.

[62] Akhavan, O, Ghaderi, E, \& Esfandiar, A. Wrapping bacteria by graphene nanosheets for isolation from environment, reactivation by sonication and inactivation by nearinfrared irradiation. J. Phys. Chem. B, (2011). , 6279-6288.

[63] Akhavan, O, \& Ghaderi, E. Escherichia coli bacteria reduce graphene oxide to bactericidal graphene in a self-limiting manner. Carbon, (2012). , 1853-1860.

[64] Zhang, D, Liu, X, \& Wang, X. Green synthesis of graphene oxide sheets decorated by silver nanoprisms and their anti-bacterial properties. J. Inorg. Biochem., (2011). , 1181-1186.

[65] Das, M. R, et al. Synthesis of silver nanoparticles in an aqueous suspension of graphene oxide sheets and its antimicrobial activity. Colloids Surf. B, (2011). , 16-22.

[66] Liu, L, Liu, J, \& Sun, D. D. Graphene oxide enwrapped Ag3PO4 composite: towards a highly efficient and stable visible-light induced photocatalyst for water purification. Catal. Sci. Technol., (2012). , 2525-2532. 